Laser Chem., 1999, Vol. 19, pp. 35-40

Reprints available directly from the publisher Photocopying permitted by license only
(C) 1999 OPA (Overseas Publishers Association) N.V.

Published by license under

the Harwood Academic Publishers imprint, part of The Gordon and Breach Publishing Group.

Printed in India.

\title{
MOLECULAR DYNAMICS INVESTIGATED BY TEMPORALLY TWO-DIMENSIONAL COHERENT RAMAN SPECTROSCOPY
}

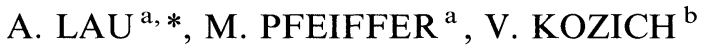 \\ and A. KUMMROW ${ }^{\mathrm{a}}$ \\ ${ }^{a}$ Max-Born-Institut für Nichtlineare Optik und Kurzzeitspektroskopie, \\ D-12489 Berlin, Germany; ${ }^{\mathrm{b}}$ Institute of Physics, Minsk, Belarus
}

(Received 7 April 1997)

\begin{abstract}
A six-wave set-up is described to determine molecular dynamics in the condensed phase. Applying two independent time delays between excitation and probe pulses additional information on the dynamics should be obtainable. We show experimentally that such investigations can be carried out with noisy light having intensity fluctuations in the femtosecond region. As first result we found a fast relaxation time in neat nitrobenzene of $100 \mathrm{fs}$, becoming even faster in mixtures with low viscosity liquids. Switching on a Raman resonance yields a longer relaxation time, which could be explained by an additional contribution by that vibration.
\end{abstract}

Keywords: Nonlinear spectroscopy; Raman; Kerr effect; fifth order nonlinearity

The knowledge of solvation dynamics is crucial for an understanding of the first steps of chemical reactions. Whereas most of the investigations are focused on electronic dephasing, a full understanding of the complex dynamics needs additional information on vibrational dynamics and nuclear motions. Access to the latter is usually obtained by applying coherent time resolved Raman techniques, based on third order susceptibilities. However, because in these experiments only one variable time delay is available $\left(\Delta t_{1}\right)$, it is in principle impossible to unambiguously distinguish between interactions which are induced by

${ }^{*}$ Corresponding author. 
rapid statistical fluctuations of the molecular transition (homogeneous linewidth) and those which are due to different environment in solutions acting on a slower time scale (inhomogeneous linewidth). Especially for intermolecular bath modes, a situation is expected just in between these two limiting cases, which is already supported by first measurements in neat $\mathrm{CS}_{2}$ [1].

In recent years two different new methods have been developed solving this problem, based on 5 th and 7 th order nonlinear susceptibilities introducing a second time variable $\Delta t_{2}$ between the probe pulse and a second vibrational excitation. The two different time dependencies allow to separate effects of homogeneous and inhomogeneous broadening [2, 3].

Here we describe the first experiment applying broadband nanosecond pulses with inherent femtosecond coherence times combined with a narrow band radiation to generate higher order coherent Raman effects. The broadband, as well as the narrow band radiation, are generated by dye-lasers pumped by the second and third harmonics of the Nd-YAG laser (Continuum, Surelight), respectively. The broadband laser is home made using a flowing dye cell and a resonator without any dispersion elements delivering Gaussian spectral distributions of 6 to $7.5 \mathrm{~nm}$ width centered at $572 \mathrm{~nm}$ (for $\mathrm{Rh} 6 \mathrm{G}$ as dye). This corresponds to coherence length of 0.3 to $0.37 \mathrm{~mm}$ or to FWHM correlation times between 90 and $112 \mathrm{fs}$ applying the Wiener Khintichine's theorem. The FWHM time was chequed by autocorrelation measurements. For the narrow band radiation we use a simple dye laser (Lasertechnik Berlin $\mathrm{GmbH}$ ) with a bandwidth of $0.1 \mathrm{~nm}$, which was tuned between 527 and $550 \mathrm{~nm}$ to provide different resonance and off resonance conditions with respect to Raman active vibrations of the investigated solvents. The broadband radiation is split into three beams and the narrow band radiation into two. All beams have app. the same intensity and are focused by a lens of $100 \mathrm{~mm}$ focal length into a $5 \mathrm{~mm}$ cell under the phasematching condition

$$
k_{\text {signal }}=k_{\mathrm{br} 3}+\left(k_{\mathrm{br} 2}-k_{\mathrm{n} 2}\right)-\left(k_{\mathrm{br} 1}-k_{\mathrm{n} 1}\right)
$$

and using the geometry described in [4]. $k_{\mathrm{bri}}$ are the $k$-vectors of the 3 broadband radiations and $k_{\mathrm{ni}}$ are those of the narrow band beams. 
The narrow band radiation can be used keeping the possibility of applying two independent time variables and simplifies the calculations considerably.

As sample, neat nitrobenzene and mixtures of nitrobenzene with toluene, acetonitrile and trifluoroethanol (TFE) are chosen. The main features in scanning the first excitation with respect to the other beams $\left(\Delta t_{1}\right)$ are shown in Figure 1. The corresponding features for a scan of the probe beam $\left(\Delta t_{2}\right)$ with respect to the others are depicted in Figure 2. For both scans peaks with FWHM of much less than 1 ps are observed. The FWHM depends on Raman resonance conditions and on concentration of nitrobenzene in the mixtures (Fig. 3). Depending on the experimental conditions (tuning $\Delta t_{1}$ for different settings of $\Delta t_{2}$ or tuning $\Delta t_{2}$ for different settings of $\Delta t_{1}$ ) different ratios between the peak and the background intensity are observed.

We find that the peak width depends less on the Raman resonance conditions, than could be expected having in mind the $T_{2}$ time for the $1345 \mathrm{~cm}^{-1}$ vibration of nitrobenzene being about $1 \mathrm{ps}$. This manifests that the measured signal is likely dominated by contributions of nuclear motions, as librations and orientational diffusion. Recently in nitrobenzene a relaxation time of $100 \mathrm{fs}$ was measured, which is of the same order of magnitude as the fast relaxation time in $\mathrm{CS}_{2}$ [5]. This value agrees well with the relaxation time of $95 \mathrm{fs}$ obtained after deconvolution of our experimental curves. This fast dephasing time of

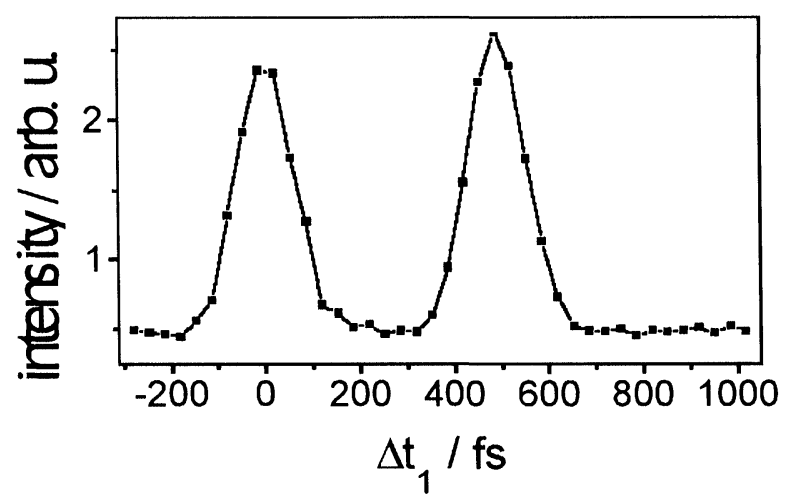

FIGURE 1 Signal intensity versus delay of the first excitation. Probe pulse delay is fixed by $500 \mathrm{fs}$. 


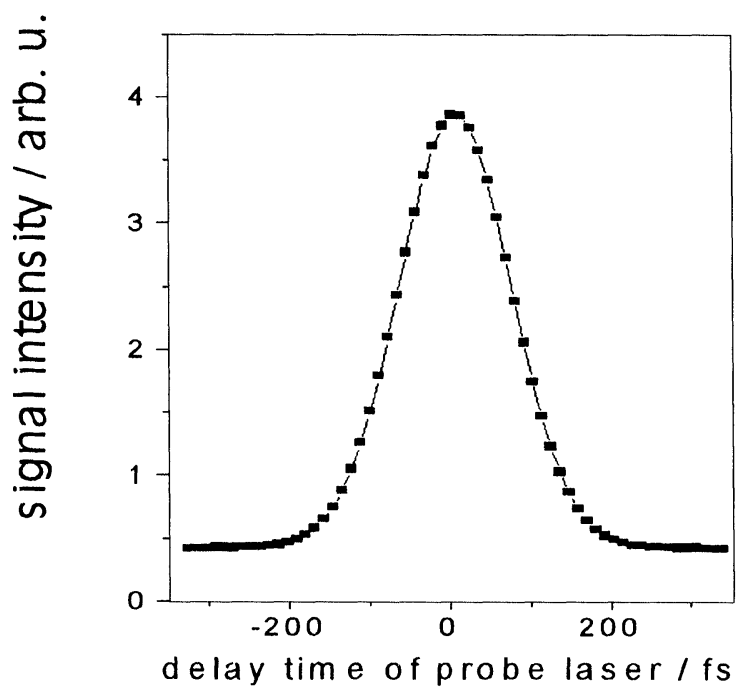

FIGURE 2 Signal intensity in dependence on the probe laser delay $\left(\Delta t_{1}=500 \mathrm{fs}\right)$.

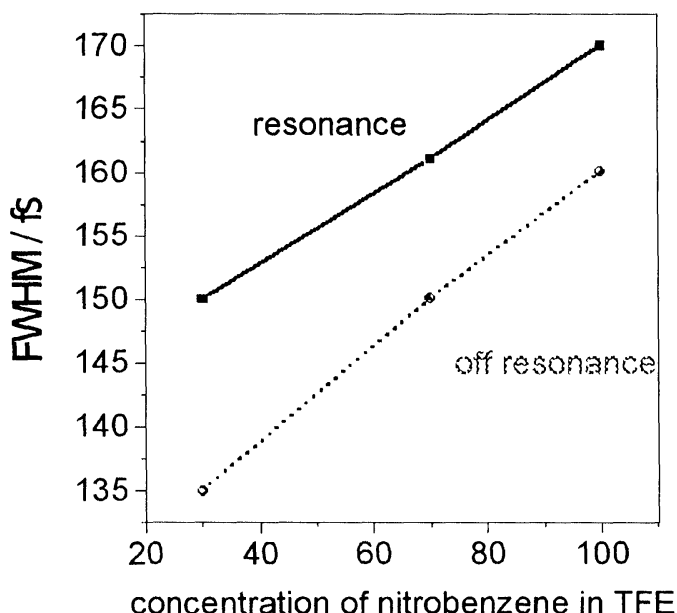

FIGURE 3 Peak's widths for a mixture of nitrobenzene in TFE under on and off Raman resonance conditions.

nitrobenzene in mixtures with TFE develops to $\tau_{\text {RES }}=82 \mathrm{fs}$ under Raman resonance excitation and to $\tau_{\mathrm{NRES}}=72 \mathrm{fs}$ under off resonance conditions for a $30 \mathrm{vol} \%$ nitrobenzene concentration. Assuming a 
relation between $\tau_{\text {NRES }}, \tau_{\text {RES }}$ and the dephasing time $T_{2}$ of the vibration as

$$
\frac{1}{\tau_{\text {NRES }}}-\frac{1}{\tau_{\text {RES }}}=\frac{2}{T_{2}},
$$

it follows a $T_{2}=1.2 \mathrm{ps}$ for neat nitrobenzene, which is near to the value we measured by Interferometric CARS.

Theoretically the peaks can be described as contributions of fifth order susceptibility around zero delay between each of the broadband components forming a Kerr-grating. The width of the peak can be modified by the additional Raman process originating from combination of the broadband laser and the anti-Stokes-shifted narrow band laser. The dependence on time delay between the broadband components is determined by the two-field correlators.

$$
<E_{\mathrm{br} 2}\left(t_{1}-\Delta t_{1}\right) E_{\mathrm{brl}^{\prime}}^{*}\left(t_{2}-\Delta t_{2}\right)>\text { and }<E_{\mathrm{br} 3}\left(t_{1}\right) E_{\mathrm{br} 1^{\prime}}^{*}\left(t_{2}-\Delta t_{2}\right)>.
$$

The fifth order peak broadened by an additional Raman contribution is expressed by a term of the type

$$
\begin{aligned}
I^{5 \text { th }}\left(\Delta t_{2}\right) \propto \cdots \int_{-\infty}^{t_{2}} d t_{1} \mathrm{e}^{-\gamma_{1}\left(t_{2}-t_{1}\right)} & <E_{\mathrm{br} 3}\left(t_{2}\right) E_{\mathrm{br} 1}^{*}\left(t_{1}-\Delta t_{2}\right)> \\
\int_{-\infty}^{s_{2}} d s_{1} \mathrm{e}^{-\gamma_{2}\left(s_{2}-s_{1}\right)} & <E_{\mathrm{br} 3}^{*}\left(s_{2}\right) E_{\mathrm{br} 1}\left(s_{1}-\Delta t_{2}\right) \\
& \Rightarrow F\left(\gamma_{1}, \Delta t_{2}\right) F\left(\gamma_{2}, \Delta t_{2}\right)
\end{aligned}
$$

representing the product of two Gaussians F. Here $\gamma_{1}$ represents a short time response, $\gamma_{2}$ gives the Raman contribution. The full expression for the intensity has to be summed up over all permutations of the field components in the two-field correlators and describes the experimental results qualitatively. For a quantitative understanding of the results other processes have to be considered.

One should note, that in the signal direction also cascading third order processes of the type of Forced Light Scattering (FLS) could evolve. Though they have a considerable phasemismatch, they can contribute to the signal or even dominate it. This is independent on whether femtosecond pulses or noisy light with femtosecond substructure is used. Therefore all these experiments have to be 
carefully analyzed taking into account the applied special conditions. Because our measured concentration dependence is in rather good agreement with the dependence of fifth order nonlinearity and the results depend strongly on the phasematching conditions, we assume in these experiments competition of third order cascade and fifth order processes with dominance of fifth order processes. In most cases the peaks can be well fitted by Gaussian curves, excluding detailed information on the librational frequency and the spectral density of the nuclar motions. Further detailed investigations are under way to solve this problem. Nevertheless our results establish a fast relaxation time of $100 \mathrm{fs}$ for neat nitrobenzene and show for the first time a decreasing relaxation time in mixtures with solvents of lower viscosity as well as an influence of exciting a vibration on the measured decay time of the nuclear motions.

\section{References}

[1] Steffen, T. and Duppen, K. (1997). J. Chem. Phys., 106, 3854.

[2] Vanden Bout, D., Muller, L. and Berg, M. (1991). Phys. Rev. Lett., 67, 3700.

[3] Tominaga, K. and Yoshihara, K. (1995). Phys. Rev. Lett., 74, 3061.

[4] Lau, A., Pfeiffer, M. and Kummrow, A. (1996). Chem. Phys. Lett., 263, 435.

[5] Lotshaw, W. T., McMorrow, D., Kalpouzos, C. and Kenney-Wallace, G. A. (1987). Chem. Phys. Letters, 136, 323. 